\title{
Proton Quark Helicity Structure via W-Boson Production in PP Collision@ Phenix
}

\author{
F. Giordano \\ for the PHENIX collaboration \\ University of Illinois at Urbana-Champaign, \\ Department of Physics, 1110 West Green Street \\ Urbana, IL 61801-3080, USA \\ fgiord@illinois.edu
}

Published 29 February 2016

\begin{abstract}
The spin structure of the proton has been long studied in the past decades, but, while the contributions to the proton spin from valence quarks is by now precisely known, large uncertainties are still affecting our knowledge of the sea quark contributions. The measurement of single-spin asymmetries of the parity violating $W$ production in pp collision allows a (quasi-)model independent access to the flavor-dependent light sea quark contributions. Being maximally parity violating, the $W$ charge can be directly realted to the quark and antiquark flavor, and in addition, moving from forward to backward rapidities with respect to the polarized proton beam direction it is possible to change the relative contributions of $\mathrm{u}$, $\mathrm{d}$, anti- $\mathrm{u}$, anti- $\mathrm{d}$ quarks, thus accessing each light-quark spin alignment with respect to the proton spin. At PHENIX, the W boson produced in pp collision at center of mass energies of about $500 \mathrm{GeV}$ is accessed via its decays into electron (muon) at central (forward) rapidities. Here the status of the analysis and the most updated results is reported.
\end{abstract}

PACS numbers: 13.88.+e, 25.75.Dw, 13.75.Cs, 13.85.-t

\section{The Proton Spin Puzzle}

Understanding how quark and gluon spins build up the proton spin has been a major quest in particle physics since the 1980s when a first measurement by the EMC collaboration published that quarks only account for $10 \%$ of the proton spin. ${ }^{1}$ Later, more precise measurements brought this contribution up to be closer to $30 \%{ }^{2}$ The proton spin structure has been so far mainly studied in Deep Inelastic Scattering (DIS) reactions (lepton + proton $\rightarrow$ lepton) accessing longitudinally polarized structure functions, which are mostly flavor insenitive. To access flavor dependent parton distribution functions (PDFs) in DIS, one needs to detect an additional hadron in

This is an Open Access article published by World Scientific Publishing Company. It is distributed under the terms of the Creative Commons Attribution 3.0 (CC-BY) License. Further distribution of this work is permitted, provided the original work is properly cited. 
the final state (Semi-Inclusive DIS, or SIDIS), which complicates the cross-section with the inclusion of fragmentation functions (FFs). Thus, the precision to which PDFs can be accessed in SIDIS is dependent on the precision to which FFs are known.

\section{2. $W$-Boson Production in Pp Collision @500 GeV}

A different type of measurement has been proposed in pp collisions to make use of the parity violating $W$ production to access directly and in a (quasi-)model independent way the light-sea-quark spin contribution to the proton spin. Being maximally parity violating, $W$ bosons can be produced only from left-handed quarks and righthanded antiquarks. Therefore, by controlling the polarization of the proton beam we can directly relate the $\mathrm{W}$ electric charge with the only possible parent quark flavor: $W^{+}$is generated by $\left(u_{L} \bar{d}_{R}\right)$, and $W^{-}$by $\left(d_{L} \bar{u}_{R}\right)$.

The quark helicity PDFs can then be accessed via longitudinal Single Spin Asymmetries (SSA) for $W s$, defined as:

$$
A_{L}^{W}=\frac{1}{P} \frac{N^{+}-N^{-}}{N^{+}+N^{-}}
$$

where $P$ is the average beam polarization and $N^{+}\left(N^{-}\right)$is the produced $W$ yield for positive (negative) proton beam helicity. The $A_{L}^{W}$ can indeed be related to the quark helicity PDFs $\Delta q(x)$ in the following way:

$$
\begin{aligned}
& A_{L}^{W^{+}}=-\frac{\Delta u\left(x_{1}\right) \Delta \bar{d}\left(x_{2}\right)-\Delta \bar{d}\left(x_{1}\right) \Delta u\left(x_{2}\right)}{u\left(x_{1}\right) \bar{d}\left(x_{2}\right)-\bar{d}\left(x_{1}\right) u\left(x_{2}\right)}, \\
& A_{L}^{W^{-}}=-\frac{\Delta d\left(x_{1}\right) \Delta \bar{u}\left(x_{2}\right)-\Delta \bar{u}\left(x_{1}\right) \Delta d\left(x_{2}\right)}{d\left(x_{1}\right) \bar{u}\left(x_{2}\right)-\bar{u}\left(x_{1}\right) d\left(x_{2}\right)},
\end{aligned}
$$

where $x_{1}\left(x_{2}\right)$ are the fractional longitudinal momenta quarks carry in the first (second) proton. Varying the rapidity $(\eta)$ with respect to the polarized beam of the measurement from forward to backward, which also means varying $x_{1}$ and $x_{2}$, we can isolate quark and antiquark contributions such that at forward rapidities the asymmetries give access to quark helicity contribution, while backward rapidities enhance the antiquark helicity contribution; in the central rapidities both quark and antiquark contribute equally.

At PHENIX, ${ }^{3} W$ bosons can be accessed via their decays into electrons in PHENIX central arms $(|\eta|<0.35)$, or into muons in PHENIX forward/backward arms $(1.2 \leq$ $\eta \leq 1.6,-2.6 \leq \eta \leq-1.2)$ :

$$
W^{ \pm} \rightarrow l^{ \pm} \nu
$$

where $l$ represents the lepton-flavor. As there are no hadrons in the final state of such decays, this measurement does not involve fragmentation functions at all. For $W s$ we have seen there is an association between forward-rapidity with quark and backward rapidity with antiquark, while the lepton decay kinematics enhances this 
simple link for the $W^{-}$case, for the $W^{+}$case the link is smeared out, so that both quark and antiquark contribute almost equally at all rapidities.

The different lepton flavor in the decay products is not the only difference between the central and the forward analyses at PHENIX. As shown in Fig. 1, only at central rapidities the Jacobian peak is clearly visible, while in the forward/backward regions the jacobian peak is suppressed for $W^{+}$and completely missing for $W^{-}$. While for both analyses the main concern is to reduce background that dilute the asymmetries and isolate leptons produced only in $W$ decays, the analysis techniques used to do so in the central and forward/backward arms at PHENIX are very different because of the presence or absence of the Jacobian peak.

\subsection{Measurements at central rapidities}

At central rapidities we access $W$ s from their decays into electron. Electron tracks and charges are determined from tracking detectors, while the energy deposit measurement in an electromagnetic calorimeter (EMCal) provide the electron $p_{T}$. For the measurement of SSA for electrons coming from $W$ decays at central rapidities one need to account for background $e^{ \pm}$coming from a) conversion from $\gamma_{\mathrm{s}}$ from $\pi^{0} / \eta$ decay, b) cosmic rays, c) background from beams, and d) Z/charm, bottom and other $\mathrm{W}$ decays. A relative isolation cut that cut out events where the energy in a cone of radius $R=0.4 \mathrm{rad}$ not from the candidate electron is $\geq 10 \%$ of the lepton energy already reduce the background contribution by a factor 10 . The remaining background is rejected by looking at the electron transverse momenta $p_{T}$ distributions and fitting the Jacobian peak. For a better extraction of the signal under the Jacobian peak, a Gaussian Regression (GR) technique has been used that uses the low $p_{T}$, background dominated spectra to define the shape of the background. Assuming a smooth curve for the background we extrapolated the shape obtained via the GR in the high $p_{T}$ region under the Jacobian peak. Once the background
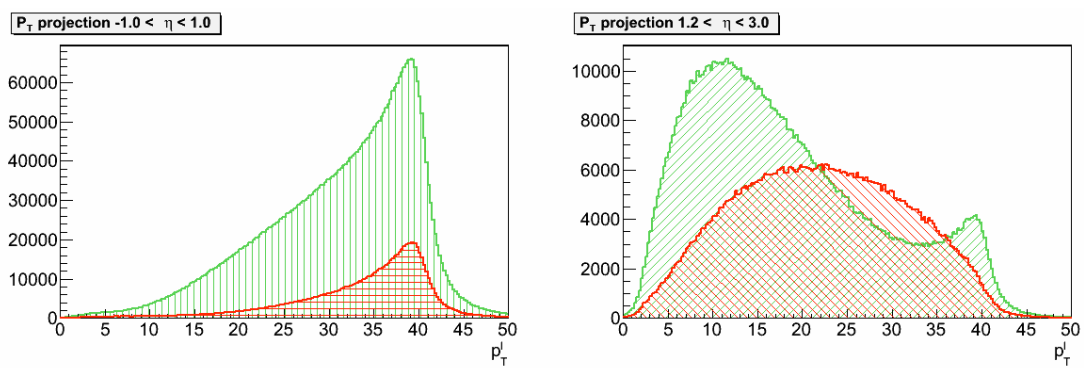

Fig. 1. Simulated transverse momentum distributions for positive (green) and negative (red) leptons from $W$ decays at central rapidities on the left and forward/backward rapidities on the right. 

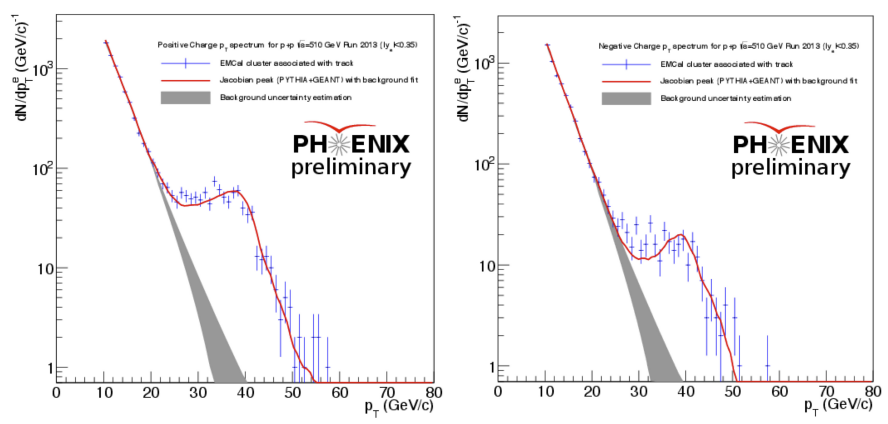

Fig. 2. Transverse momentum distributions for positive (left) and negative (right) electrons at central rapidities. The grey area gives the background shape extrated via a Gaussian Regression technique, and the red like is the fit of the background plus the Jacobian peak of the signal.

shape under the Jacobian peak is known, the whole $p_{T}$ distribution is fit including the background shape and a signal shape for the Jacobian peak, after it has been smeared in momentum, (see Fig. 2). After such fit, we have found that for $p_{T}>30$ $\mathrm{GeV} 95 \%(81 \%)$ of the candidate electrons are electrons from decays of $W^{+}\left(W^{-}\right)$.

The SSA after correction for dilution due to the remaining background under the Jacobian peak will be presented together with the forward/bakward asymmetries in section 2.3 .

\subsection{Measurements at forward rapidities}

In its forward/backward arms PHENIX can detect the muons coming from $W$ decays. Initially, low- $p_{T}$ muon backgrounds were heavily dominating the PHENIX forward muon triggers. To improve the trigger efficiency, an upgrade of PHENIX forward muon arms has been implemented. This consisted in including in the muon trigger information from the MuonTracker detectors, a set of 3 tracking stations of cathode strip chambers already existing, and then to add 2 new stations of Resistive Plate Chamber detectors per arm. While PHENIX has preliminary results for data collected in 2011 and 2012, only in 2013 for the first time the muon forward trigger upgrade was fully implemented. Here preliminary results for the forward muon SSA from $W$ collected in 2013 run are presented.

As for the central rapidities, a number of backgrounds contribute and they can be summarized in 2 groups:

- muonic background, or muons from open-heavy flavor decay, quarkonia, DrellYan,

- hadronic background, where either: a) real hadrons punch-through the forward PHENIX absorbers and are reconstructed as muons, or b) hadrons decay in the 
forward arms and the original plus one decay tracks are reconstructed together as a single high- $p_{T}$ muon.

Most of these backgrounds concentrate in the low- $p_{T}$ region $\left(p_{T}<15 \mathrm{GeV}\right)$, but because of reconstructed momentum smearing they contribute largely also at higher $p_{T}$, where muons from $W$ decays are supposed to dominate. As the Jacobian peak is suppressed or completely missing for muons from $W$ in the forward/backward rapidities, it cannot be used to reduce the background and extract a signal-tobackground ratio, needed to correct the measured muon yields.

To reduce the contributions from background, a likelihood method has been implemented that determines the likelihood of an event to be a $W$ event (its Wness). The Wness is defined as:

$$
W n e s s=\frac{\lambda_{s i g}}{\lambda_{s i g}+\lambda_{b g}},
$$

where $\lambda_{s i g}\left(\lambda_{b g}\right)$ is the signal (background) likelihood, based on probability densities $(\mathcal{P} D F \mathrm{~s})$ from up to 9 variables describing the kinematic and geometry of the event. The shapes for the signal $\mathcal{P} D F$ s have been extracted from Monte Carlo simulation, as $W$ decay kinematics is well known, while the background $\mathcal{P} D F$ s have been extracted from the background-dominated data (where initially the signal is $<1 \%$ ). A cut of Wness $>0.99$ has been found to maximize the (statistical+systematic) Figure-of-Merit for the final extracted asymmetries, thus this is the cut we applied on the data sample.

From the event sample that survive the Wness $>0.99$ cut selection we can evaluate muons from $W$ yields to build the SSA, but, as a non-negligible background still contaminate the sample, an extended, unbinned maximum likelihood (EUML) fit is performed to determined the remaining signal-to-background ratio which will be used to correct the asymmetries for background dilution. The EUML fit is performed in 2 dimensions: the pesudorapidity $\eta$ and the effective track bending $d w_{23}$
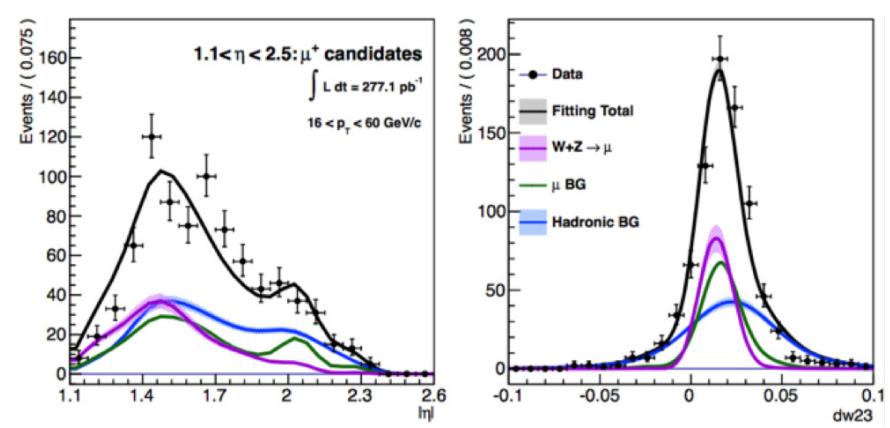

Fig. 3. EUML fit results to determine the signal-to-background ratio. The points represent the data, the fit results are given in violet for the signal and in blue for the hadronic background. The muonic background contribution (in green) is fixed by simulations. 


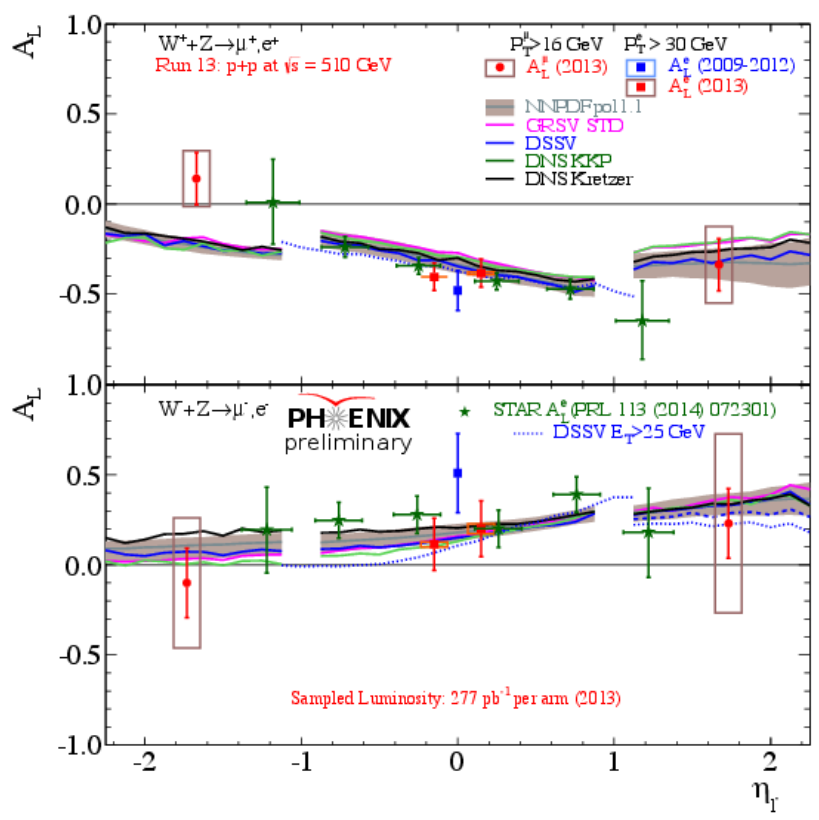

Fig. 4. Single-Spin-Asymmetries for electron (muon) from $W$ versus the rapidity $\eta$ in the central (forward/backward) arm as measured in data from 2013 PHENIX run in red and in previous runs in blue. Top panel is for $W^{+}$, bottom panel for $W^{-}$. PHENIX results are here compared with STAR asymmetries in green, and predictions from global analyses.

between the two outermost track chambers; both these variables are independent from the ones used in the Wness determination. The Likelihood is written as:

$$
\mathcal{L}(\theta \mid X) \equiv \frac{n^{N} e^{-N}}{N !} \prod_{x_{i} \in X}^{N} \sum_{c} \frac{n_{c}}{n} p_{c}\left(x_{i}\right),
$$

where $\theta$ gives the 3 parameters $n_{c}=n_{\text {sig }}, n_{\mu}, n_{\text {had }}$ for the signal, muon background and hadronic background counts respectively, and $n=\sum n_{c}$. Here, $p_{c}\left(x_{i}\right)$ stands for the $\mathcal{P} D F$ of the $c$-th parameter for the 2 variables $x_{i}=\eta, d w_{23}$. The product is extended to all the $N$ events in the data sample.

In the EUML fit the shape of muon backgrounds is fixed by simulation, as their sources are well known, while their absolute contribution is defined via a scale factor that we extract by comparing measured and simulated dimuon spectra. The fit then determines the signal (muons from $W$ ) and hadronic background contribution to the data sample. For the signal the $\mathcal{P} D F$ shapes are taken again from simulation, the hadronic background $\mathcal{P} D F$ shapes instead come directly from the low-Wness region of the data itself, which is hadronic-background dominated. An example for the results of the EUML fit is given in Fig. 3. The extracted signal-to-background ratio ranges from $30 \%$ to $50 \%$ depending on the muon charge and PHENIX arm. 


\subsection{Single-Spin-Asymmetries}

Figure 4 shows preliminary single-spin-asymmetries for leptons generated in $W$ decays in the central (electrons) and forward/backward (muons) rapidities for 2013 PHENIX run in red. The asymmetries are consistent with theory expectations, and are compatible with the global analyses predictions (also shown in Fig. 4) within the uncertainties, except the negative rapidity point for $W^{+}$, which is about 2.5 sigma away from predictions. The analyses are being finalized to be published in the near future, and PHENIX results will be then ready to be included in PDFs global analyses for a better estimation of flavor-dependent light sea quark PDFs.

\section{References}

1. EMC Collab. (J. Ashman et al.), Phys. Lett. B206, 364 (1988).

2. HERMES Collab. (A. Airapetian et al.), Phys. Rev. D75, 012007 (2007).

3. Phenix Collab. (K. Adcox et al.), Nucl. Instrum. Methods A499, 469 (2003). 